05

\title{
Исследование прочностных характеристик акрилонитрилбутадиенстирол пластика при динамических нагрузках
}

\author{
(C) А.А. Чеврычкина, ${ }^{1,2}$ А.Д. Евстифреев, ${ }^{1}$ Г.А. Волков ${ }^{1,2}$ \\ ${ }^{1}$ Институт проблем машиноведения РАН, \\ 199178 Санкт-Петербург, Россия \\ ${ }^{2}$ Санкт-Петербургский государственный университет, \\ 199034 Санкт-Петербург, Россия \\ e-mail: anastasiia.che@gmail.com
}

(Поступило в Редакцию 12 июля 2017 г.)

Проведены экспериментальные исследования по определению механических свойств аддитивного материала, изготовленного из акрилонитрилбутадиенстирол пластика на 3D принтере, в квазистатическом и динамическом режимах. С помощью критерия инкубационного времени построена прочностная кривая, описывающая нелинейную зависимость критического напряжения от скорости деформации. Определено значение характерного инкубационного времени $\tau$ сопоставлением теоретической кривой с экспериментальными данными.

DOI: 10.21883/JTF.2018.03.45596.2430

\section{Введение}

В настоящее время все большее внимание уделяется методам 3D печати [1]. Хотя эти методы уже много лет применяются в промышленности, разработка относительно дешевых потребительских 3D принтеров придала аддитивным технологиям производства новый импульс. Сегодня многие люди ожидают отличного будущего для 3D печати. Некоторые эксперты считают, что эти методы могут привести к новой промышленной революции и в будущем наши здания будут напечатаны на строительной площадке.

Несмотря на весь потенциал 3D печати вообще и 3D печати в строительной отрасли, в частности, еще необходимо исследовать свойства напечатанного материала, чтобы его можно было бы применить в строительной индустрии. Важно знать, как себя поведет материал в различных условиях эксплуатации. Критическое напряжение, при котором материал разрушается, является одной из основных характеристик материала. Известно, что для многих материалов, например бетона и горных пород, при увеличении скорости деформации критическое напряжение растет [2-4]. В 1974 г. Н.А. Златиным и его коллегами [5] экспериментально был получен эффект наличия „динамической ветви“ при динамических разрушениях. А именно была зафиксирована возможность выдерживать материалами кратковременные нагрузки, многократно превосходящие их статический предел прочности, данный эффект хорошо описывается критерием инкубационного времени [6-8].

Основными целями настоящей работы являются определение прочностных характеристик продуктов 3D печати в условиях экстремальных нагрузок и анализ полученных эффектов с использованием структурно-временного подхода.

\section{1. Развитие динамических методов исследования материалов}

Хорошо известно, что многие материалы при динамических и квазистатических нагрузках ведут себя поразному. Ранние работы в этой области принадлежат B. Hopkison [9] и W. Manson [10], они использовали падающий наконечник для получения импульса растяжения. Гинс (Ginns) [11], используя пружинный механизм для приложения внезапной нагрузки и тензодатчика для измерения напряжения, был одним из первых, кто попытался напрямую записать динамическую кривую напряжения - деформации. Позже Brouw и Vincent [12] с помощью маятниковой ударной машины и пьезоэлектрических кристаллов для измерения напряжений получили кривые напряжения - деформации непосредственно на экране осциллографа.

До 1940 г. измерение напряжения всегда включало один из двух методов. Напряжение вычислялось из соображений теории распространения упругих волн и поэтому было ограничено упругой областью или использовался метод с полумеханической измерительной головкой напряжения, что почти всегда сопровождалось большими колебаниями напряжения. Однако в 1940 г. Fanning и Bassett [13] разработали методику измерения ударных деформаций с помощью тензорезисторов с электрическим сопротивлением, а в 1948 г. Brown и Edmonds [14] применили ударную маятниковую машину типа Брауна и Винсента. Это позволило фиксировать импульсы напряжения растяжения во времени, которые не искажали колебания напряжения, возникающие в измерительной головке.

Большинство исследователей, помимо Hopkinson и Manson, игнорировали влияние распространения волны напряжения в образце, считая, что напряжение, измеренное на конце образца, эквивалентно напряжению во всем 
образце. Гэст (Guest) [15] в 1930 г. пришел к выводу, что, когда скорость удара достаточно высока, распространение и отражение волн напряжения в испытательной установке, если их игнорировать, могут привести к значительным ошибкам в результатах. Это было показано в 1948 г. в работах Clark и Duwez [16], в которых применена теория распространения пластических волн к испытаниям на растяжение.

Clark и Wood [17] работе 1949 г. описали устройство нового типа растягивающей машины, в которой сила приложена пневматически и достигает максимального значения в течении $5 \mu \mathrm{s}$. Такой тип нагружения они называли „быстрым“, под которым они подразумевали динамическое нагружение со скоростями, при которых эффект распространения волны напряжения в аппарате пренебрежимо мал. Это отличается от ударного нагружения, когда волновые эффекты должны быть приняты во внимание.

Campbell and Duby [18] в 1956 г. были первыми, кто выполнил ударную нагрузку на аппарате, спроектированном специально для анализа результатом в терминах волнового распространения. Они смогли получить значение предела текучести мягкой стали, когда время воздействия $25 \mu \mathrm{s}$ и максимальная скорость деформации порядка $1000 \mathrm{~s}^{-1}$.

В настоящее время наиболее востребованной экспериментальной методикой в мире для исследования динамического поведения материалов при скоростях деформаций порядка $10^{2}-10^{4} \mathrm{~s}^{-1}$ является метод Кольского с использованием разрезного стержня Гопкинсона (РСГ) или его модификации. Изначально этот метод был предназначен только для испытания на одноосное сжатие, метод постоянно совершенствовался и на сегодняшний день существуют сжимающий, растягивающий, крутильный, сдвиговой и двуосный варианты метода РСГ.

В настоящей работе исследования динамических характеристик аддитивного материала проводились на башенном копре Instron CEAST 9350 при скорости деформации порядка $10^{2}-10^{3} \mathrm{~s}^{-1}$.

\section{2. Материал и методика исследования}

В настоящей работе рассматривается аддитивный материал, изготовленный из акрилонитрилбутадиоенстирол (ABS) пластика на 3D принтере. Образцы соответствуют стандарту ISO 8256 методу А, внешний вид и геометрия образцов приведены на рис. 1. Печать образцов проводилась послойно, толщина слоя $0.06 \mathrm{~mm}$.

Испытания проведены для 10 различных скоростей деформации от $2 \cdot 10^{-5}$ до $2 \cdot 10^{3} \mathrm{~s}^{-1}$. Для каждой скорости деформации было проведено 5 испытаний.

\section{1. Квазистатические свойства}

Квазистатические исследования проводились на установке Shimadzu AG-50kNXD, оборудованной экстензометром, при скорости деформации $2 \cdot 10^{-3}$ и $2 \cdot 10^{-5}$.

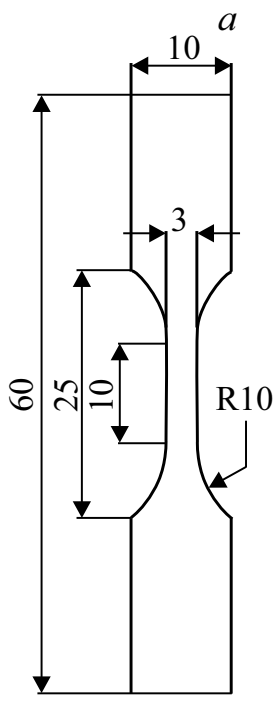

$b$

Рис. 1. $a$ - геометрия и размеры образца (толщина образца $1 \mathrm{~mm}), b-$ внешний вид испытуемых образцов.

Свойства материалов

\begin{tabular}{c|c|c}
\hline Свойства & Образец & Филамент \\
\hline$E, \mathrm{MPa}$ & 1700 & $1700-2930$ \\
$\sigma_{c}, \mathrm{MPa}$ & 36.5 & $26-47$
\end{tabular}

Значение модуля Юнга определялось как угол наклона линейно-упругой части кривой напряжение - деформации, т.е. $E=d \sigma / d \varepsilon$. Предел прочности определялся как максимальная сила к площади сечения образца. Значения модуля упругости и предела прочности приведены в таблице. Стоит отметить, что полученные в опытах величины модуля Юнга и предела прочности для напечатанных образцов находятся в интервале значений, указанных производителем, для исходного материала.

\section{2. Динамические свойства}

Динамические исследования производились на башенном копре Instron Ceast 9350 с функцией дополнительной энергии, скорость ударника от 0.77 до $24 \mathrm{~m} / \mathrm{s}$.

Образец помещается таким образом, что нижний конец помещен в захваты, по которым приходится удар с заданной скоростью ударника. Верхняя часть закреплена на неподвижной стойке, на которой находится пьезоэлектрический датчик силы. Типичный профиль силы от времени для трех разных скоростей деформации 180 , 455 и $20351 / \mathrm{s}$ для проведенных испытаний приведен на рис. 2. Напряжение линейно возрастает до своего максимального значения, затем значение силы немного уменьшается и держится постоянным некоторое время, в это время в образце развиваются процессы, которые ведут к разрушению. За значение динамического предела прочности бралось отношение максимального значения 


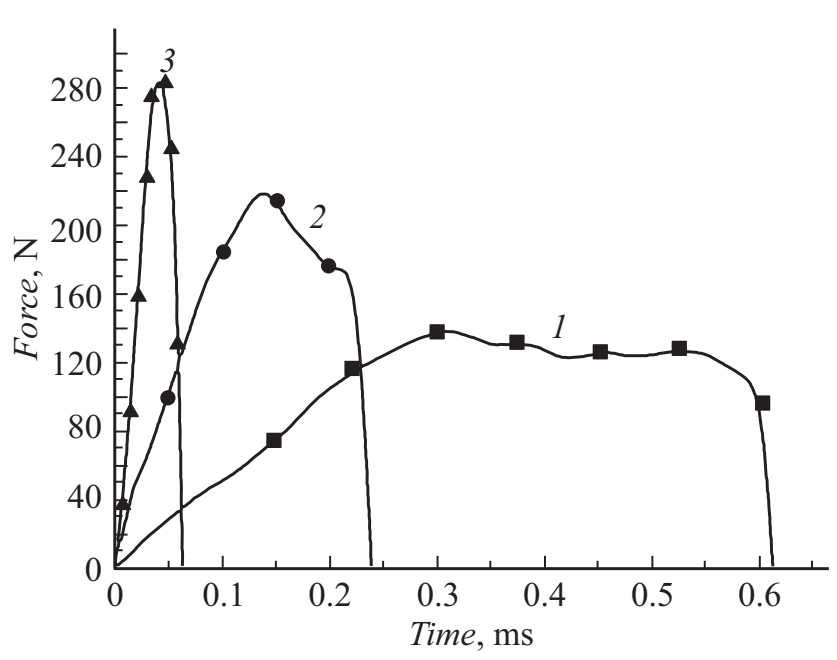

Рис. 2. Типичный профиль силы от времени для трех разных скоростей деформации: $1-180,2-455,3-2035$ 1/s.

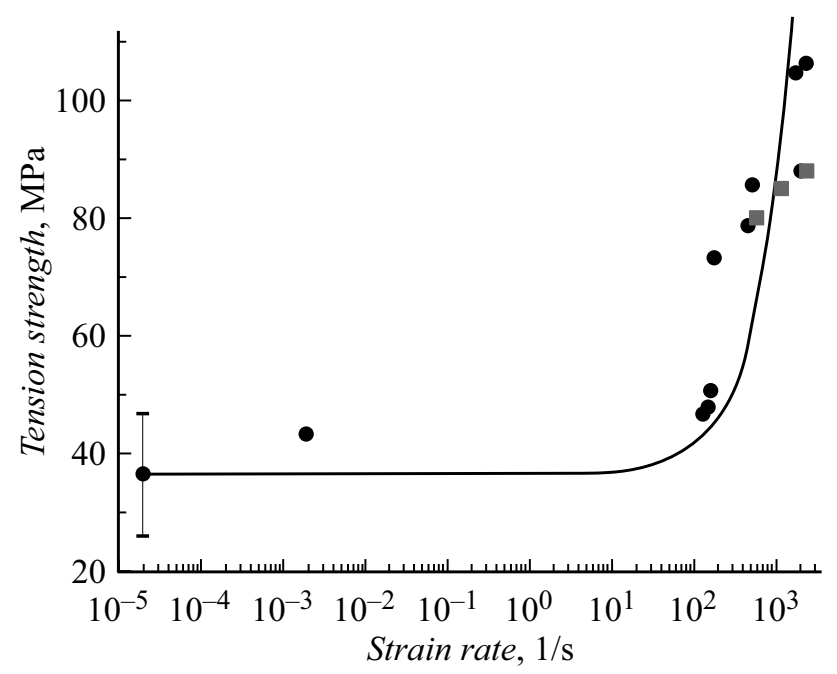

Pис. 3. Зависимость критического напряжения от скорости деформации. Кривая построена по критерию (1) при $\sigma_{c}=36.5 \mathrm{MPa}, \tau=57 \mu \mathrm{s}$, черные круглые точки - экспериментальные данные для аддитивного материала, серые квадраты - экспериментальные данные для филамента из работы [19].

силы к начальной площади сечения образца. На рис. 3 приведены усредненные результаты испытаний для каждого режима, также приведены данные из работы [19] для исходного ABS-пластика, из которого печатались образцы. Как в случае статического нагружения, так и для динамического нагружения значение предельного напряжения для напечатанного материала мало отличается от исходного материала. Сравнение данных для напечатанного и исходного материалов позволяют считать, что при высоком качестве печати прочностные свойства меняются слабо. Выполненные испытания показали, что предел прочности напечатанных образцов из ABS-пластика существенно зависит от скорости дефор- мации, значения критического напряжения нелинейно возрастают с увеличением скорости деформации.

\section{3. Критерий инкубационного времени}

Критическое напряжение принято считать постоянной материала при статическом нагружении. В динамических испытаниях, как было отмечено выше, критическое напряжение зависит от скорости деформаций, способа приложения нагрузки и не является константой. В связи с этим возникает проблема, что же считать динамической прочностью материала, и как предсказывать предельные прочностные характеристики для динамических воздействий произвольной формы. Ключом к решению данной задачи может служить структурно-временной подход с использованием инкубационного времени [6-8]. В рамках этого подхода считается, что разрушение наступает тогда, когда текущее напряжение превышает статический предел прочности на протяжении некоторого временно́го промежутка, длительность которого определяется формой разрушающего импульса и значением инкубационного времени.

Критерий инкубационного времени можно выразить следующей формулой $[6,7]$ :

$$
\int_{t-\tau}^{t} \sigma(s) d s \leq \sigma_{c} \tau,
$$

где $\sigma_{c}$ - статическая прочность на растяжение, $\tau-$ инкубационное время материала, $\sigma(s)$ - временно́й профиль нагружающего импульса.

Результаты проведенных испытаний показывают, что напряжения возрастают линейно до своего максимального значения (рис. 2), потому зависимость напряжений в образце от времени вплоть до момента разрушения может быть описана следующей функцией:

$$
\sigma(t)=E \dot{\varepsilon} t H(t)
$$

где $H(t)$ - функция Хэвисайда, $E-$ модуль упругости материала, $\dot{\varepsilon}$ - скорость деформации. Другими словами, в данной экспериментальной схеме было реализовано жесткое нагружение образца, т. е. $\dot{\varepsilon}=$ const. Подстановка выражения для временного профиля напряжений (2) в критерий (1) позволяет определить уровень критических напряжений для определенного значения $\dot{\varepsilon}$. Таким образом, кривая скоростной зависимости прочности может быть рассчитана аналитически для любого диапазона скоростей деформации образца.

Критерий разрушения (1), используемый в структурно-временно́м подходе, содержит два определяющих параметра: статическую прочность и инкубационное время материала, которое можно условно считать динамическим параметром прочности, поскольку в динамике именно от его значения в первую очередь зависит величина критических напряжений в момент разрушения. 
Инкубационное время можно интерпретировать, как характерное время, необходимое для подготовки изучаемого процесса разрушения в образце. В настоящее время нет стандартных экспериментальных методов для прямого измерения значений $\tau$, так как для этого требуется высокоточная техника. Некоторое оптимальное значение инкубационного времени может быть определено сопоставлением аналитической кривой, рассчитанной по критерию (1) с экспериментальными данными. В качестве условия наилучшего совпадения можно использовать, например, метод наименьших квадратов.

На рис. 3 приведены результаты экспериментов и расчетная кривая для ABS-пластика в квазистатических и динамических режимах работы. Кривая соответствует расчетам по формуле (1) при $\sigma_{c}=36.5 \mathrm{MPa}, \tau=57 \mu \mathrm{s}$.

Результаты расчетов показывают, что кривая, полученная с помощью критерия инкубационного времени, не только довольно точно описывает экспериментальные данные во всем диапазоне скоростей нагружения, реализованных в ходе испытаний, но и предсказывает возможные значения прочности материалов в неисследованных областях по шкале скорости деформации. Используя данный подход, можно на качественном уровне подбирать материалы под индивидуальные условия эксплуатации.

\section{Заключение}

Проведенные экспериментальные исследования по измерению критического напряжения аддитивного материала, изготовленного из ABS-пластика на 3D принтере, в квазистатическом и динамическом режимах показали, что статическая прочность и модуль Юнга напечатанных образцов соответствует значениям, указанным производителем исходного филамента. В ходе динамических испытаний была измерена величина критического напряжения в материале для скоростей деформации в диапазоне $10^{-5}$ до $10^{3} \mathrm{~s}^{-1}$, и полученная скоростная зависимость прочности была описана с помощью критерия инкубационного времени. В ходе расчетов было определено значение характерного инкубационного времени $\tau=57 \mu \mathrm{s}$, которое также может быть использовано для предсказания разрушения напечатанного материала в случае динамических ударных импульсов произвольной формы.

Также стоит отметить, что проведенная в настоящей работе серия статических динамических испытаний, с последующей обработкой результатов с помощью критерий инкубационного времени, может рассматриваться, как некая стандартизированная методика, позволяющая определить скоростную зависимость критических напряжений в любом диапазоне скоростей деформации. Для этого необходимо определить параметры материала в квазистатическом режиме нагружения и сделать контрольные замеры в динамическом режиме. Используемый башенный копер позволяет определить критическое напряжение для исследуемых образцов при скоростях деформации порядка $10^{2}-10^{3} \mathrm{~s}^{-1}$, для более высоких скоростей деформации значения критического напряжения предсказываются по критерию инкубационного времени.

Работа выполнена при финансовой поддержке: Волков Г.А. и Чеврычкина А.А. выполнили разд. 1,2 гранта РНФ (17-11-01053), Естифеев А.Д. выполнил разд. 3 - гранта РФФИ (16-31-60003 мол_а_дк). Исследования проведены с использованием оборудования ресурсного центра Научного парка СПбГУ „Исследование экстремальных состояний материалов и конструкций/Scientific research were performed at the Research park of St.Petersburg State University The study of extreme states of materials and constructions".

\section{Список литературы}

[1] Campbell T. et al. Could 3D Printing Change the World? 2011.

[2] Goldsmith W. et al. // Rock. Mech. Min. Sci. Geomech. 1976. Vol. 13. P. 303.

[3] Howe S., Goldsmith W., Sackman J. // Experimental Mechanics. 1974. Vol. 14. P. 337.

[4] Petrov Y.V., Smirnov I.V., Utkin A.A. // Mech. Solids. 2010. Vol. 45. N 1. P. 476.

[5] Златин Н.А., Мочалов С.М., Пугачев Г.С., Брагов А.М. // ФTT. 1974. Т. 16. Вып. 6. С. 1752.

[6] Petrov Y.V, Utkin A.A. // Mater Sci. 1989. Vol. 25. N 2. P. 153.

[7] Petrov Y.V. On „quantum“ nature of dynamic fracture of brittle solids. Dokl Akad Nauk USSR. 1991. Vol. 321. P. 66.

[8] Петров Ю.В., Ситникова Е.В. // ЖТФ. 2004. Т. 74. Вып. 1. C. 58-61. (Petrov Yu.V., Sitnikova E.V. // Technical Physics. 2004. Vol. 49. N 1. P. 57.)

[9] Hopkinson B. // Proc. Roy. Soc. 1905. Vol. 74. Series A. P. 498.

[10] Manson W. // Proc. Instn. Mech. Engrs. 1934. Vol. W. 128. P. 409.

[11] Ginns D.W. // J. Inst. Met. 1937. Vol. 61. P. 61.

[12] Brown A.F.C., Vincent N.D.G. // Proc. Instn. Mech. Engrs. 1941. Vol. 145. P. 126.

[13] Fanning R., Bassett W. // J. Appl. Mech. 1940. Vol. 7. P. 24.

[14] Brown A.F.C., Edmonds R. // Proc. Instn. Mech. Engrs. 1948. Vol. 159. P. 11.

[15] Guest J.J. // Proc. Instn. Mech. Engrs. 1930. P. 1273.

[16] Clark D.S., Duwez P.E. // J. Appl. Mech. 1948. Vol. 15. P. 243.

[17] Clark D.S., Wood D.S. // Proc. Amer. Soc. Test. Mater. 1949. Vol. 49. P. 717.

[18] Campbell J.D., Duby J. // Proc. Roy. Soc. 1956. Series A. Vol. 236. P. 24.

[19] Yin Z.N., Wang T.J. // Mater. Sci. Engineer. A. 2010. Vol. 527. N 6. P. 1461. 